\title{
Nonclassical Pseudospectral Method for a Class of Singular Boundary Value Problems Arising in Physiology
}

\author{
A. Alipanah
}

Department of Mathematics, University of Kurdistan, Sanandaj, Iran

\begin{abstract}
In this paper, nonclassical pseudospectral method is presented for solution of a classof nonlinear singular boundary value problems arising in physiology. Properties of non-classical pseudospectral method are presented. These properties are utilizeto reduce the computation of singular boundary value problems to system of equations. Numerical method is tested for its efficiency by considering two examples from physiology
\end{abstract}

Keywords Boundary Value Problems, Singular Points, Nonclassical Pseudospectral Method, Physiology

\section{Introduction}

There is considerable literature on the numerical treatment of singular boundary value problems[1-4]. The numerical treatment of singular boundary value problems has always been far from trivial, because of the singularity at some points. Consider a class of nonlinear singular boundary value problems of the following form $[4,9]$

$$
\begin{gathered}
y^{\prime \prime}(x)+\frac{m}{x} y^{\prime}(x)=f(x, y), \quad 0 \leq x \leq 1 \\
y^{\prime}(0)=0 \\
\alpha y(1)+\beta y^{\prime}(1)=\gamma
\end{gathered}
$$

where $\alpha, \beta$ and $\gamma$ are real numbers and we assume that $\mathrm{f}(\mathrm{x}$, y) $\epsilon\{L 2[0,1] \times R\}$ is continuous, $\frac{\partial f}{\partial y}$ exists and continuous, and $\frac{\partial \mathrm{f}}{\partial \mathrm{y}} \geq 0, \forall \mathrm{x} \in[0,1]$. For the case $\mathrm{m}=2, \alpha=\gamma$ and $\beta=1$ the existence and uniqueness of the solution (1)-(3) has been given in[18].

This work is based on the Michaelis-Menten kinetics[5] for the steady state oxygendiffusion in spherical cells, in which

$$
f(x, y)=f(y)=\frac{n y(x)}{y(x)+k}, k>0, n>0
$$

A similar problem arise in the study of the distribution of heat sources in the humanhead[6-8] in which

$$
f(x, y)=f(y)=-n e^{-n k y(x)}, k>0, n>0
$$

Point wise bounds and uniqueness results are given in[6] for this problem with $\mathrm{f}(\mathrm{x}, \mathrm{y})$ of the form given by (4) and (5). Pandy and Singh[4] have used finite difference method (FD) based on uniform mesh, Kanth and Bhattacharya[9] usedcubic spline method of order $\mathrm{O}(\mathrm{h} 4)$ for solving (1)-(3) approximately. The objective of this paper is to use

* Corresponding author:

A.Alipanah@uok.ac.ir (A. Alipanah)

Published online at http://journal.sapub.org/am

Copyright (C 2012 Scientific \& Academic Publishing. All Rights Reserved nonclassical pseudospectral method for approximation singular boundary value problem (1)-(3). Theoretical studies and numerical experiences have confirmed that for problems with smooth solution pseudospectral methods converge faster than other methods[10]. The idea of employing nonclassical weight functions first has been used by Shizgal[11] for solving the Boltzmann equations, Planck equations, and Shizgal and Heli Chen[12] used these basis for the solution eigenvalues and eigenfunction of Schrodinger equation.

\section{Nonclassical Pseudospctral Discretization Method}

The nonclassical pseudospectral methods[11,12,20] expand the function $f \in L^{2}[a, b] b y$ using weighted interpolations of degree $\mathrm{N}$ of the form[11,12]

$$
f^{N}(x) \cong P_{N}(x)=\sum_{j=0}^{N} \frac{W(x)}{W\left(x_{j}\right)} L_{j}(x) f\left(x_{j}\right), x \in[a, b]
$$

where $x_{j}, j=0,1, \ldots, N$ are a set of distinct collocation points on the interval[a,b], $\mathrm{W}(\mathrm{x})$ is a positive weight function, and $\mathrm{L}_{\mathrm{j}}(\mathrm{x})$ are a set of Lagrange interpolating polynomials that satisfy $L_{j}(x k)=\delta_{j k}$, i.e

$$
L_{j}(x)=\prod_{\substack{k=0 \\ k \neq j}}^{N}\left(\frac{x-x_{k}}{x_{j}-x_{k}}\right)
$$

\subsection{Collocation Points}

Consider the orthogonal polynomials $\mathrm{p}_{\mathrm{n}}(\mathrm{x})$ with respect to some weight function $\mathrm{w}(\mathrm{x})$ on the interval $[\mathrm{a}, \mathrm{b}]$, that is

$$
\int_{a}^{b} w(x) p_{n}(x) p_{m}(x) d x=\delta_{m, n} .
$$

The polynomials satisfy a three-term recurrence relation[13]

$$
p_{k+1}(x)=\left(x-\alpha_{k}\right) p_{k}(x)-\beta_{k-1} p_{k-1}(x), k=0,1,2
$$




$$
p_{-1}(x)=0, \quad p_{0}(x)=1
$$

where the coefficients satisfy the inner product formulae [13]

$$
\begin{array}{r}
\alpha_{k}=\frac{\int_{a}^{b} x w(x) p_{k}^{2}(x) d x}{\int_{a}^{b} w(x) p_{k}^{2}(x) d x}, k=0,1,2 \\
\beta_{0}=\int_{a}^{b} w(x) p_{0}^{2}(x) d x, \beta_{k}=\frac{\int_{a}^{b} w(x) p_{k}^{2}(x) d x}{\int_{a}^{b} w(x) p_{k-1}^{2}(x) d x}
\end{array}
$$

The collocation points $x_{j}$ and weights $\mathrm{w}_{\mathrm{j}}$ may be determined by the method outlined by Golub and Welsch[14]. The approach is based on determining the eigenvalues and normalized eigenvectors of a modified tri-diagonal Jacobi matrix,

$$
J=\left[\begin{array}{ccccc}
\alpha_{0} & \sqrt{\beta_{1}} & & & \\
\sqrt{\beta_{1}} & \alpha_{1} & \sqrt{\beta_{2}} & & \\
& \sqrt{\beta_{2}} & \alpha_{2} & \sqrt{\beta_{3}} & \\
& \ddots & \ddots & \ddots & \\
& & \sqrt{\beta_{N-1}} & \alpha_{N-1} & \sqrt{\beta_{N}^{*}} \\
& & & \sqrt{\beta_{N}^{*}} & \alpha_{N}^{*}
\end{array}\right]
$$

where $\beta_{N}^{*}, \alpha_{N}^{*}$ are obtained from the solution of the linear system of equations

$$
\left[\begin{array}{ll}
p_{N(a)} & p_{N-1}(a) \\
p_{N}(b) & p_{N-1}(b)
\end{array}\right]\left[\begin{array}{l}
\alpha_{N}^{*} \\
\beta_{N}^{*}
\end{array}\right]=\left[\begin{array}{l}
a p_{N}(a) \\
b p_{N}(b)
\end{array}\right]
$$

The collocation points $\mathrm{x}_{\mathrm{j}}$, including the end points, are determined as the eigenvalues of $\mathrm{J}$, and weights $\mathrm{w}_{\mathrm{j}}$ obtained in terms of the first components $v_{1 j}$ of then ormalized eigenvector $\mathrm{v}$ as follows:

$$
\mathrm{w}_{\mathrm{j}}=\beta_{0}\left(\mathrm{v}_{1 \mathrm{j}}\right)^{2}
$$

The integration of a function, $\mathrm{f}(\mathrm{x})$ may be approximated by the Gauss-Lobattoquadrature rule as

$$
\int_{a}^{b} w(x) f(x) d x \cong \sum_{j=0}^{N} w_{j} f\left(x_{j}\right)
$$

Where $\mathrm{w}_{\mathrm{j}}$ are the weights associated with the collocation points $\mathrm{x}_{\mathrm{j}}, \mathrm{j}=0,1, \ldots, \mathrm{N}$.

\subsection{Computation of Differential Matrices}

A number of algorithms have been proposed for generating pseudo spectral differential matrices numerically. In this section, the algorithm developed by Welfert[13] is employed and summarized as follows:

(1) Calculate the diagonal elements:

(a) Initialize $D_{\mathrm{kk}}^{(\mathrm{m})}$ using the equation

$$
D_{k k}^{(p)}=\frac{W^{(p)}\left(x_{k}\right)}{W\left(x_{k}\right)}, 0 \leq k \leq N, 0 \leq p \leq m
$$

(b) Update $\mathrm{D}_{\mathrm{kk}}^{(\mathrm{m})}$ recursively using the equation

$$
\begin{gathered}
D_{k k}^{(p)} \leftarrow D_{k k}^{(p)}+\frac{p}{x_{k}-x_{j}} D_{k k}^{(p)}, 0 \leq k \leq N, 0 \leq p \leq m, \\
0 \leq j \neq k \leq N
\end{gathered}
$$

(2) Calculate the off-diagonal elements:

(a) Initialize the off-diagonal elements using the equation

$$
D_{k j}^{(0)}=0,0 \leq k \leq N, 0 \leq j \neq k \leq N
$$

(b) Update $D_{\mathrm{kj}}^{(\mathrm{m})}$ using the following equation

$$
\begin{gathered}
D_{k j}^{(p)}=\frac{p}{x_{k}-x_{j}}\left[D_{k j}^{(p-1)}-D_{k k}^{(p-1)}\right], \\
0 \leq k \leq N, 1 \leq p \leq m, 0 \leq j \neq k \leq N .
\end{gathered}
$$

For practical numbers of collocation points, the above algorithm is generally sufficient.

\section{Discretization of Singular Boundary Value Problem}

In this section, we solve the singular boundary value problem (1)-(3) by using nonclassicalpseudospectral method. From Eq. (1) we have that boundary valueproblem is singular at point $x=0$. Now we collocate Eq. (1) in the points $\mathrm{x}_{\mathrm{r}}, \mathrm{r}=1,2, \ldots, \mathrm{N}-1$ which at this points Eq. (1) is not singular. For this purpose we first substitute Eq. (7) for $\mathrm{y}(\mathrm{x})$ in Eqs. (1)-(3), i.e

$$
\begin{gathered}
\sum_{j=0}^{N} y_{j}\left[\frac{W(x)}{W\left(x_{j}\right)} L_{j}(x)\right]^{(2)}+\frac{m}{x} \sum_{j=0}^{N} y_{j}\left[\frac{W(x)}{W\left(x_{j}\right)} L_{j}(x)\right]^{(1)} \\
=f\left(x, \sum_{j=0}^{N} y_{j} \frac{W(x)}{W\left(x_{j}\right)} L_{j}(x)\right) \\
\mathrm{y}^{\prime}(0)=0 \\
\alpha \mathrm{y}(1)+\beta \mathrm{y}^{\prime}(1)=\gamma
\end{gathered}
$$

Now collocate the equation (15) at $\mathrm{x}=\mathrm{x}_{\mathrm{r}}, \mathrm{r}=1,2, \ldots, \mathrm{N}-1$ and by using the differential matrices obtained in section 2 , the above equations can be written as follows:

$$
\begin{gathered}
\sum_{j=0}^{N} D_{r j}^{(2)} y_{j}+\frac{m}{x_{r}} \sum_{j=0}^{N} D_{r j}^{(1)} y_{j}=f\left(x_{r}, x_{r}\right), r=1,2, \cdots, N-1(18) \\
\sum_{j=0}^{\mathrm{N}} \mathrm{D}_{0 j}^{(1)} \mathrm{y}_{\mathrm{j}}=0 \\
\alpha \mathrm{y}_{\mathrm{N}}+\beta \sum_{\mathrm{j}=0}^{\mathrm{N}} \mathrm{D}_{\mathrm{Nj}}^{(1)} \mathrm{y}_{\mathrm{j}}=\gamma
\end{gathered}
$$

Now equations (18)-(20) are a system of nonlinear equations that can be solved by Newton iterative method for the resulting nonlinear systems.

\section{Numerical Examples}

In this section, we have used the method presented for different weighted functions $\mathrm{w}(\mathrm{x})$ and $\mathrm{W}(\mathrm{x})$ given in Table 1, and on two physical model examples: (i) oxygen diffusion; (ii) nonlinear heat conduction model of human head. These problems already been studied by Asaithambi and Goodman[19], Pandy and singh[4], Kanthand Bhattacharya[9]. The numerical results show that present method approximates the solution very well and computational time is less than other methods.

Table 1. Different $w(x)$ and $\mathrm{W}(x)$ on $[0,1]$

\begin{tabular}{|c|c|c|}
\hline Cases & $w(x)$ & $\mathrm{W}(x)$ \\
\hline 1 & 1 & 1 \\
2 & $1+0.5 \cos (x)$ & $\mathrm{e}^{-2 x}$ \\
3 & $\frac{1}{1+x}$ & $1+0.5 \cos (x)$ \\
4 & $e^{-x^{2}}$ & $\mathrm{e}^{-x}$ \\
\hline
\end{tabular}


Table 2. Numerical results for $\mathrm{N}=10$ and different cases for Example 1

\begin{tabular}{|c|c|c|c|c|}
\hline$x_{i}$ & Case 1 & Case 2 & Case 3 & Case 4 \\
\hline 0.0 & 0.8284832903 & 0.8284832915 & 0.8284832901 & 0.8284832903 \\
0.1 & 0.8297060924 & 0.8297060935 & 0.8297060921 & 0.8297060924 \\
0.2 & 0.8333747335 & 0.8333747346 & 0.8333747333 & 0.8333747335 \\
0.3 & 0.8394899139 & 0.8394899154 & 0.8394899137 & 0.8394899139 \\
0.4 & 0.8480527849 & 0.8480527859 & 0.8480527847 & 0.8480527850 \\
0.5 & 0.8590649271 & 0.8590649282 & 0.8590649269 & 0.8590649271 \\
0.6 & 0.8725283199 & 0.8725283212 & 0.8725283197 & 0.8725283199 \\
0.7 & 0.8884453056 & 0.8884453066 & 0.8884453053 & 0.8884453056 \\
0.8 & 0.9068185480 & 0.9068185492 & 0.9068185478 & 0.9068185480 \\
0.9 & 0.9276509883 & 0.9276509895 & 0.9276509881 & 0.9276509883 \\
1.0 & 0.9509457984 & 00.9509457996 & 0.9509457982 & 0.9509457985 \\
\hline
\end{tabular}

Table 3. Numerical results by other methods

\begin{tabular}{|c|c|c|}
\hline xi & Cubic spline [9] method for $h=\frac{1}{60}$ & Panndy and singh [4] \\
\hline 0.0 & 0.8284832730 & 0.8284831497 \\
0.1 & 0.8297060752 & 0.8297060742 \\
0.2 & 0.8333747169 & 0.8333747157 \\
0.3 & 0.8394898186 & 0.8394898966 \\
0.4 & 0.8480527704 & 0.8480527648 \\
0.5 & 0.8590649140 & 0.8590649116 \\
0.6 & 0.8725283084 & 0.8725283056 \\
0.7 & 0.8884452959 & 0.8884452928 \\
0.8 & 0.9068185402 & 0.9068185369 \\
0.9 & 0.9276509825 & 0.9276509791 \\
1.0 & 0.9509457946 & 0.9509457914 \\
\hline
\end{tabular}

Table 4. Numerical results for $\alpha=\beta=1, \gamma=0$ and for $\mathrm{N}=10$.

\begin{tabular}{|c|c|c|c|c|c|}
\hline$x_{i}$ & Case 1 & Case 2 & Case 3 & Case 4 & Method in [9] \\
\hline 0.0 & 0.3675168151 & 0.3675168056 & 0.3675168157 & 0.3675168146 & 0.367517980 \\
0.1 & 0.3663623292 & 0.3663623199 & 0.3663623299 & 0.3663623287 & 0.366363492 \\
0.2 & 0.3628940661 & 0.3628940569 & 0.3628940667 & 0.3628940655 & 0.362895222 \\
0.3 & 0.3570975457 & 0.3570975355 & 0.3570975463 & 0.3570975451 & 0.357098689 \\
0.4 & 0.3489484206 & 0.3489484114 & 0.3489484212 & 0.3489484201 & 0.348949546 \\
0.5 & 0.3384121487 & 0.3384121390 & 0.3384121494 & 0.3384121482 & 0.338413250 \\
0.6 & 0.3254435224 & 0.3254435122 & 0.3254435231 & 0.3254435218 & 0.325444592 \\
0.7 & 0.3099860402 & 0.3099860304 & 0.3099860409 & 0.3099860396 & 0.309987070 \\
0.8 & 0.2919711030 & 0.2919710928 & 0.2919711037 & 0.2919711024 & 0.291972083 \\
0.9 & 0.2713170101 & 0.2713169998 & 0.2713170108 & 0.2713170095 & 0.271317928 \\
1.0 & 0.2479277233 & 0.2479277127 & 0.2479277240 & 0.2479277227 & 0.247928565 \\
\hline
\end{tabular}

Table 5. Numerical results for $\alpha=0.1, \beta=1$, and $\gamma=0$, and for $\mathrm{N}=10$

\begin{tabular}{|c|c|c|c|c|c|}
\hline$x_{i}$ & Case 1 & Case 2 & Case 3 & Case 4 & Method in [9] \\
\hline 0.0 & 1.1470390193 & 1.1470390105 & 1.1470390196 & 1.1470420530 & 1.1470410835 \\
0.1 & 1.1465096424 & 1.1465096336 & 1.1465096426 & 1.1465126739 & 1.1465117057 \\
0.2 & 1.1449205020 & 1.1449204933 & 1.1449205023 & 1.1449235440 & 1.1449225634 \\
0.3 & 1.1422685635 & 1.1422685546 & 1.1422685638 & 1.1422716181 & 1.1422706215 \\
0.4 & 1.1385487483 & 1.1385487395 & 1.1385487486 & 1.1385517959 & 1.1385508014 \\
0.5 & 1.1337539033 & 1.1337538944 & 1.1337539036 & 1.1337569825 & 1.1337559499 \\
0.6 & 1.1278747567 & 1.1278747476 & 1.1278747569 & 1.1278778561 & 1.1278767950 \\
0.7 & 1.1208998607 & 1.1208998517 & 1.1208998610 & 1.1209029636 & 1.1209018887 \\
0.8 & 1.1128155198 & 1.1128155107 & 1.1128155201 & 1.1128186642 & 1.1128175352 \\
0.9 & 1.1036057040 & 1.1036056948 & 1.1036057042 & 1.1036088683 & 1.1036077042 \\
1.0 & 1.0932519451 & 1.0932519454 & 1.0932519454 & 1.0932551446 & 1.0932539271 \\
\hline
\end{tabular}

\subsection{Example 1}

Consider the oxygen diffusion corresponding Eqs. (1)-(4) with $\mathrm{n}=0.76129, \mathrm{k}=0.03119, \alpha=\gamma=5$ and $\beta=1$. Numerical results for cases given in Table 1 , be given in Table 2, and numerical results with other methods be given in Table 3.

\subsection{Example 2}

The second example is of nonlinear heat conduction model of the human head, which correspond to Eqs. (1)-(3) and (5) with $\mathrm{n}=1 ; \mathrm{k}=1$. Numerical results by our method are given in Tables 4 and 6 . We have performed calculations 
for the following two cases:

(i) $\alpha=\beta=1$ and $\gamma=0$,

(ii $\alpha=0.1, \beta=1$, and $\gamma=0$.

Table 4, shows numerical results for case (i) and all weighted functions given in Table 1 . Also in Table 5, numerical results for case (ii) $\alpha=0.1, \beta=1$, and $\gamma=0$ together with results of cubic spline[9] of Kanth and Bhattacharya are given.

\section{Conclusions}

The nonclassical pseudo spectral method has been used to solve a class of singular boundary value problems arising in physiology. The main advantage of the presented approach is that it is possible to select arbitrary weight functions for the generation of the orthogonal polynomials. The cubic spline[9] and the method given in[4] have the disadvantage that the number of system is large and also the approximation is not good. The numerical results show that the proposed method is very accurate and needs less computational efforts respect to cubic spline[9] and the finite difference[4] methods.

\section{REFERENCES}

[1] Chawla, M. M., and Katti, C. P., 1982, Finite difference methods and their convergence for a class of singular two-point boundary value problems, Numer. Math, 39, 341-350

[2] Chawla, M. M., Mckee, S., and Shaw, G., 1986, Order $\mathrm{h}^{2}$ method for a singular two-point boundary value problem, BIT, $26,326-381$

[3] El-Gebeily, M. A., and Abu-Zaid, I. T., 1998, On a finite difference method for singular two-point boundary value problems, IMA J. Numer. Anal, 18, 179-190

[4] Pandey, R. K., and Singh, R. K., 2004, On the convergence of a finite difference method for a classof singular boundary value problems arising in physiology, Journal of Computational Applied Mathematics, 166, 553-564

[5] McElwain, D. L. S, 1978, A re-examination of oxygen diffusion in a spherical cell with Michaelis Menten oxygen uptake kinetics, J. Theor. Biol, 71, 255-263

[6] Duggan, R. C., and Goodman, A. M., 1986, Point wise bounds for a nonlinear heat conduction modelof the human head, Bull. Math. Biol, 48, 229-236

[7] Flesch, U., 1975, The distribution of heat sources in the human head: A theoretical considerationJ. Theor. Biol, 54, 285-287

[8] Garner, J. B., and Shivaji, R., 1990, Diffusion problems with mixed nonlinear boundary condition,J. Math. Anal.Appl, 148 $422-430$

[9] Ravi Kanth, A. S. V., and Bhattacharya, V., Cubic spline for a class of nonlinear singularboundary value problems arising in physiology, Journal of Computational Applied Mathematics, 2005

[10] Canuto, C., Hussaini, M. Y., Quarteroni, A., and Zang, T. A., Spectral Methods in Fluid Dynamics, Springer-Verlag, New York, 1987

[11] Shizgal, B., 1981, A Gaussian quadrature procedure for use in the solution of the Boltzmann equationand related problems, Journal of Computational Physic 41, 309-328

[12] Shizgal, B., and Chen, H., 1996, The quadrature discretization method (QDM) in the solution of the Shrödinger equation with non-classical basis functions, Journal of Chemical Physics 104(11), 4137-4150

[13] Gautschi, W., 1985, Orthogonal polynomials-constructive Theory and applications, Journal of Computational Applied Mathematics, 12/13, 61-75

[14] Golub, G. H., and Welsch, J. H., 1996, Calculation of Gauss quadrature rules, Mathematics of Computation, 23, 221-230

[15] Elnagar, G. N., and Kazemi, M. A., 2004, Numerical periodic optimal control: Apseudo-spectral Fourier approach, Numerical Functional Analysis and Optimization, 25, 707-724

[16] Elnagar, G. N., and Kazemi, M. A., and Razzaghi, M., 1995, Thepseudo-spectral Legendre method for discretizing optimal control problems, IEEE Tran. Automat. Cont, 40(10), 1793-1796

[17] Welfert, B. D., 1997, Generation of pseudo-spectral differentiation matrices, SIAM Journal of Numerical Analysis, 24, $1640-1657$

[18] Hiltmann, P., Lory, P., 1983, On oxygen diffusion in a spherical cell with Michaelis-Menten oxygen uptake kinetics, Bull. Math. Biol, 45,661-664

[19] Asaithambi, N. S., and Goodman, J. B., 1989, Point wise bounds for a class of singular diffusion problems in physiology, App. Math. Comput, 30, 215-222

[20] A. Alipanah, M. Razzaghi and M. Dehghan, 2007, Nonclassical pseudospectral method for the solution of brachistochrone problem, Chaos, Solitons and Fractals, 34, 1622-1628 